\title{
POTENTIAL FLUCTUATIONS IN HgSe:Fe,Ga - A NUMERICAL STUDY*
}

\author{
P. Sobkowicz, Z. Wilamowski and J. Kossut \\ Institute of Physics, Polish Academy of Sciences \\ Al. Lotników 32/46, 02-668 Warszawa, Poland
}

\begin{abstract}
We study spatial correlation of donor charges in $\mathrm{HgSe}: \mathrm{Fe}, \mathrm{Ga}$ using a few simple quantities (mean electrostatic energy of various donor sites, fluctuations of the electrostatic potential). The study uses Monte Carlo simulation techniques. We are able to indicate general trends in the behavior of the correlation when proportion of correlating $\left(\mathrm{Fe}^{3+}\right)$ and noncorrelating $(\mathrm{Ga})$ charged centers in the sample is changed.
\end{abstract}

PACS numbers: $71.55 .-\mathrm{i}$

\section{Introduction}

Despite the fact that spatial correlations of positions of charges on dopants in a mixed valence regime in $\mathrm{HgSe}: \mathrm{Fe}$ and similar materials are studied since considerable time [1-4], our knowledge of the correlation process is far from being complete. The reason is that correlation is a complicated, many-body phenomenon, occurring in a system with internal disorder. The general description is very difficult, and analytical models work reasonably well only in certain limiting cases. For example, for weak correlation one can use an analytical short-range correlation model [3,5]. Another possibility is the impurity self screening approach [4]. For strong correlation, however, only numerical simulations are practical $[2,6]$. These are unfortunately time consuming and require big computers.

The description of the correlation is usually based on only a few functional characteristics of the system: the pair correlation function $g(R)$, the structure factor $S(q)$, the density of states (DOS) [4]. Some of the models are capable if yielding only some of these quantities (e.g., the short-range correlation model gives $g(R)$ and $S(q)$, but not DOS).

As a result, it is rather difficult to obtain an insight into general properties of the spatial correlation process. We would like to concentrate on a few quantities characterizing the correlation, which would make a comparison between different systems possible. In this work we present results of a series of Monte Carlo simulations aimed at a description of the correlation in a system containing both

*This work is supported in part by the Committee for Scientific Research under grant no. 204839101. 
correlating and noncorrelating donor species (e.g., Fe and $\mathrm{Ga}$ in $\mathrm{HgSe}$ [7]). To this end we investigate the average values and the amplitude of fluctuations of the electrostatic potential energy at various lattice sites (respectively, the first and second moment of DOS). Both quantities are important: the changes of the mean value of the potential govern the Fermi energy of the system, whereas the fluctuations determine scattering of carriers [8].

\section{Definitions}

The model Monte Carlo (MC) system studied in this work may be described as follows: of 1000 sites distributed randomly within a $\mathrm{MC}$ cell 400 are assumed to be neutral, 600 - to be positively charged. In the latter group some are considered fixed, i.e., they do not change position during the simulation process, remaining in the initial, random configuration. The rest of the charged sites may take part in the correlation process, through exchange of positions with neutral sites. The three types of sites correspond to various donor sites in the $\mathrm{HgSe}: \mathrm{Fe}, \mathrm{Ga}$ system: neutral sites $-\mathrm{Fe}^{2+}$ ions, mobile charges $-\mathrm{Fe}^{3+}$ sites, fixed charges $-\mathrm{Ga}^{+}$ sites. We study the behavior of the system for various proportions of fixed and mobile charges. By keeping the number of all charges constant, we are able to concentrate on effects of correlations in the system. The details of the Monte Carlo simulation technique may be found in our earlier publications [9]. Our simulations are performed for $T=0$.

We study the electrostatical potential distribution (which is directly related to the single particle density of states) on various donor sites in the system: neutral, charged - mobile and fixed. Our work concentrates on the mean potential value $E_{\text {mean }}$ and potential fluctuations, given by the second moment of the distribution of potential values, $\sigma$. It should be noted here that the mean energy may be obtained from two-particle correlation functions $g(R)$, whereas the fluctuations require knowledge of higher-order correlations.

\section{Results}

The main results of our study are presented in Figs. 1 and 2. Figure 1 shows the behavior of the mean potential energy at various donor sites $\left(\mathrm{Fe}^{2+}, \mathrm{Ga}\right.$, and $\mathrm{Fe}^{3+}$ ) as a function of the number of the neutral sites. In addition, the mean energy of all charged sites $\left(\mathrm{Ga}\right.$ and $\mathrm{Fe}^{3+}$ ) is also plotted. The continuous line presents mean energy of all charged sites calculated from analytical short-range model [2]. The difference between mean energies of charged and neutral sites is a sign of the presence of correlations in the system. For a truly random system the values would be the same for all types of sites. An inset shows the behavior of DOS configuration with 300 mobile/ 300 fixed/400 neutral sites. Figure 2 presents the dependence of the fluctuations of the potential energies, $\sigma$, as a function of the number of fixed charges in the system.

For both studied quantities, the behavior for low and high content of the fixed sites is interesting. We can see that an admixture of a small number of the fixed sites practically does not change neither the mean energies nor the fluctuations. This is due to the fact that when there are only a few fixed charges they 

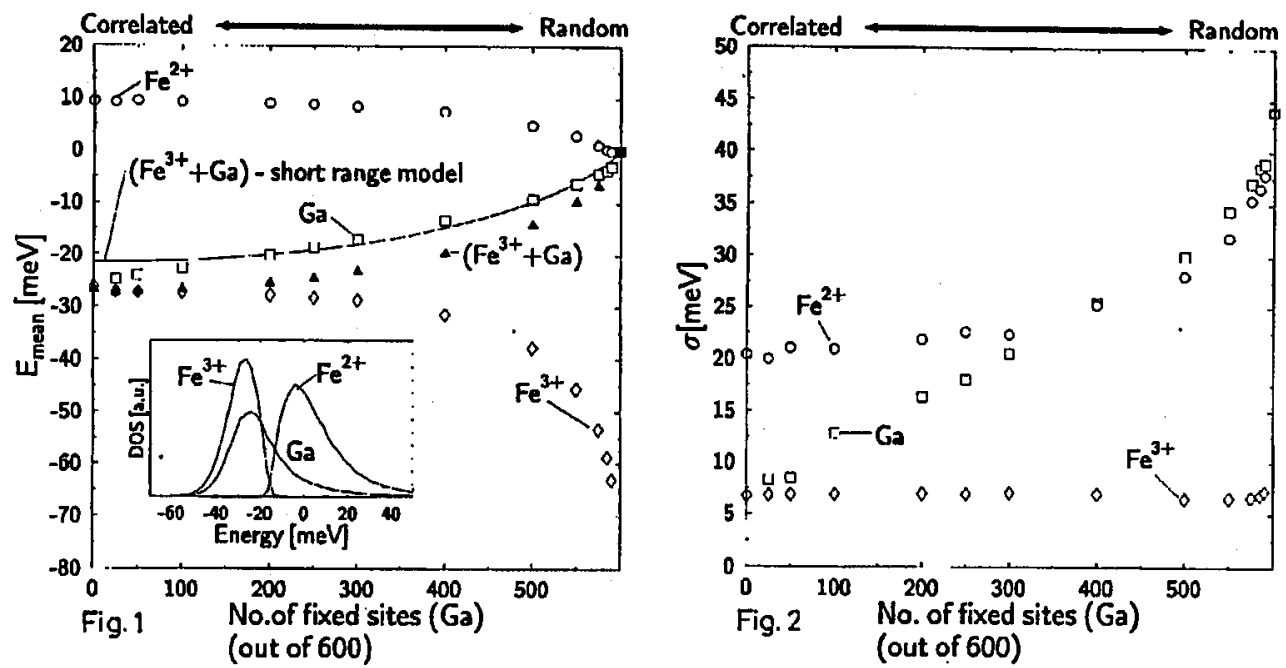

Fig. 1. Mean electrostatic potential energy at various impurity sites $\left(\mathrm{Fe}^{2+}\right.$ - neutral, $\mathrm{Ga}$ - charged, fixed, $\mathrm{Fe}^{3+}$ - charged, mobile). The triangles denote the mean energy of all charged sites $\left(\mathrm{Ga}\right.$ and $\mathrm{Fe}^{3+}$ ). The solid line presents the mean energy of all charged sites as calculated from analytical short-range model. The system has a constant number of charged sites - 600 (with the number of fixed sites taken as an independent variable) and 400 neutral sites. The inset shows potential distribution for various types of sites for a system with 300 mobile and 300 fixed charges. Numerical value of energies calculated for total doping density $(\mathrm{Fe}+\mathrm{Ga})$ of $1.5 \times 10^{19} \mathrm{~cm}^{-3}$ in $\mathrm{HgSe}$.

Fig. 2. The second moment of distribution of potential energies, $\sigma$, at various types of impurity sites.

are usually far from each other so that their interactions are small. The mobile charges adjust their positions taking the presence of the fixed charges into account, and the ordering in the system is almost as good as it is in the case of only mobile charges/neutral sites. This description is supported by MC results of the pair correlation functions. Our results thus confirm assumptions used earlier in the analytical short-range model for a mixed $\mathrm{HgSe}: \mathrm{Fe}, \mathrm{Ga}[2,7]$. Another interesting result is that although the average energy of the charged mobile $\mathrm{Fe}^{3+}$ sites decreases with their decreasing number, the corresponding $\sigma$ remains practically unchanged.

We turn now to the behavior of the system when there are only a few mobile charges, the rest remaining fixed and randomly distributed. As may be seen from Fig. 2 even a small number of mobile charges decreases significantly both the mean energy of charged sites and fluctuations of the potential. This means that the effect of correlation is very strong, even when the number of particles taking active part in correlations is small. It should be noted here that in the limit when all charges are fixed and distributed randomly the average energy is equal to zero (compensated by the uniform neutralizing charge of the conduction band electrons). At the same 
time $\sigma$ calculated from simulations agrees with analytical formula for a random system [5].

\section{Conclusions}

In this work we propose a simple set of numerical parameters which are at the same time significant physically and intuitively understandable, and which allow us to compare the properties of different systems where spatial correlations of charges occur. The quantities described in this work are related to the distribution of electrostatic potential energy at impurity sites in semiconductor. The splitting of energy distributions for various types of sites is a characteristic sign of the presence of correlations, and diminishes with increasing proportion of random fixed charges. There is a continuous transition from a system where all charges take part in the correlations to a system where there are no correlations and charges are distributed randomly in space. We show that even a small number of correlating charges may significantly lower the potential fluctuations and mean energy of the charged sites.

\section{References}

[1] B.I. Shklovskii, A.L. Efros, Electronic Properties of Doped Semiconductors, Springer Series in Solid-State Sciences, Vol. 45, Springer, Berlin 1984.

[2] Z. Wilamowski, K. Świątek, T. Dietl, J. Kossut, Solid State Commun. 73, 833 (1990).

[3] Z. Wilamowski, Acta Phys. Pol. A 77, 133 (1990).

[4] J. Kossut, Z. Wilamowski, T. Dietl, K. Świątek, in: The Physics of Semiconductors, Proc. 20th Int. Conf., Eds. E.M. Anastassakis, J.D. Joannopoulos, World Scientific, Singapore 1990, p. 613.

[5] Z. Wilamowski, W. Jantsch, G. Ostermayer, J. Kossut, in: 16 ICDS, Bethlehem 1991, Eds. G. Davies, G.G. Deleo, M. Stavola, Materials Science Forum, Vol. 83-87, Trans. Tech. Publ., Zürich 1992, p. 805.

[6] P. Sobkowicz, Z. Wilamowski, J. Kossut, to be published in J. Phys., Condens. Matter, 1993.

[7] C. Skierbiszewski, Z. Wilamowski, T. Suski, J. Kossut, B. Witkowska, Semiçond. Sci. Technol. 8, S40 (1993); C. Skierbiszewski, PhD. Thesis, unpublished, 1992.

[8] I.Y. Yanchev, B.G. Arnaudov, S.K. Evtimova, J. Phys. C, Solid State Phys. 12, L765 (1979).

[9] P. Sobkowicz, Z. Wilamowski, J. Kossut, Semicond. Sci. Technol. 7, 1155 (1992). 